\title{
ABELIAN RANK OF NORMAL TORSION-FREE FINITE INDEX SUBGROUPS OF POLYHEDRAL GROUPS
}

BY

YOUN W. LEE

\begin{abstract}
Suppose that $P$ is a convex polyhedron in the hyperbolic 3-space with finite volume and $P$ has integer $(>1)$ submultiples of $\pi$ as dihedral angles. We prove that if the rank of the abelianization of a normal torsion-free finite index subgroup of the polyhedral group $G$ associated to $P$ is one, then $P$ has exactly one ideal vertex of type $(2,2,2,2)$ and $G$ has an index two subgroup which does not contain any one of the four standard generators of the stabilizer of the ideal vertex.
\end{abstract}

1. Introduction. Polyhedral groups provide easy examples of discrete subgroups of $\operatorname{PSL}(2, C)=\operatorname{SL}(2, C) /$ center. $\operatorname{PSL}(2, C)$ acts on the hyperbolic 3-space, $\mathbf{H}^{3}=$ $\{(x+y i+z j): x, y, z \in \mathbf{R}$ and $z>0\}$, as linear fractional transformations and it is the full group of orientation preserving isometries of $\mathbf{H}^{3}$.

Given a convex polyhedron $P$ in $\mathbf{H}^{3}$ with dihedral angles which are integer $(>1)$ submultiples of $\pi$, we define the polyhedral group $G$ associated to $P$ as the subgroup of orientation preserving isometries in the reflection group $G^{*}$ generated by the reflections of $\mathbf{H}^{3}$ in the faces of $P$. We always assume that $P$ is of finite volume. $P$ is a fundamental domain of the action of $G^{*}$ on $\mathbf{H}^{3} . P$ is topologically a closed 3-dimensional ball with finitely many points removed from the boundary corresponding to the ideal vertices of $P$. The polyhedra of this type are classified in [1 and 11]. In the simplest case, there are 32 distinct tetrahedral groups [11] (see also $\S 3$ of this paper for the list).

A discrete torsion-free subgroup of $\operatorname{PSL}(2, C)$ produces an orientable hyperbolic 3-manifold (Riemannian manifold of constant curvature -1) as its orbit space and every complete orientable hyperbolic 3-manifold arises this way. A torsion-free finite index (t.f.i.) subgroup of a polyhedral group is obviously discrete and it produces a hyperbolic 3-manifold of finite volume. Some of the early examples of hyperbolic 3 -manifolds correspond to subgroups of polyhedral groups [9, 13]. The complement of the figure-eight knot corresponds to a torsion-free index 12 subgroup of the tetrahedral group $T^{19}$ [11] (see also $\S 3$ of this paper). The complement of the Whitehead link corresponds to a torsion-free index 12 subgroup of the pentahedral group (PSL(2, Z(i)) or Picard group) [13]. See [4] for more examples.

We study in this paper the rank of abelianization ( = first homology) of normal t.f.i. subgroups of polyhedral groups. This is motivated by asking whether a polyhedral group contains a knot or link group. Our method depends on the

Received by the editors January 3, 1984 and, in revised form, October 23, 1984

1980 Mathermatics Subject Classification. Primary 57S30.

1985 American Mathematical Society $0002-9947 / 85 \$ 1.00+\$ .25$ per page 
normality and does not extend to t.f.i. subgroups. If $N$ is a t.f.i. subgroup of a polyhedral group associated to a polyhedron with $k$ ideal vertices, then the orbit space $\mathbf{H}^{3} / N$ has at least $k$ cusps by Theorem 2 (see also Remark 2). This implies that $H_{1}\left(\mathbf{H}^{3} / N ; \mathbf{Z}\right) \cong H_{1}(N ; \mathbf{Z})$ has at least rank $k$. If $N$ is further isomorphic to a link group, then by [12], the link has at least $k$ components. Therefore, a knot group may be found only when $k=1$ and the figure-eight knot is such an example. But Theorem 1 of this paper implies that a knot group cannot occur as a normal subgroup unless the polyhedron is of special type which is described next.

A polyhedron $P$ (or the associated polyhedral group $G$ ) is defined to be exceptional if (i) $P$ has exactly one ideal vertex of the type $(2,2,2,2)$ and (ii) $G$ has an index 2 subgroup which does not contain any one of the four standard generators (rotations) of the stabilizer of the ideal vertex.

There are four types of ideal vertices: $(2,2,2,2),(2,3,6),(2,4,4)$ and $(3,3,3)$ [11], where $\pi$ divided by an integer is the dihedral angle between two adjacent faces sharing the vertex. Regular vertices are of types $(2,2, n), n>1,(2,3,3),(2,3,4)$ and $(2,3,5)$.

We will simply call the rank of the abelianization of a group the rank of the group in this paper.

THEOREM 1. If a polyhedral group G contains a normal t.f.i. subgroup of rank 1, then $G$ is exceptional.

The next theorem is well known, but a proof is given to be complete.

THEOREM 2. Suppose that $N_{2}$ is a finite index subgroup of $N_{1}$, where $N_{1}$ acts simplicially on a simplicial complex $X$. Then (dimension of $\left.H_{*}\left(X / N_{1} ; \mathbf{R}\right)\right) \leq($ dimension of $\mathrm{H}_{*}\left(\mathrm{X} / \mathrm{N}_{2} ; \mathbf{R}\right)$ ).

REMARK 1. No tetrahedral group is exceptional. Therefore, the figure-eight knot group is contained in the tetrahedral group $T^{19}$ not as a normal subgroup. Theorem 1 is not true if "polyhedral group" is replaced with "reflection group".

REMARK 2. Let $G$ be the polyhedral group associated to a polyhedron $P$ with $k$ ideal vertices. The action of $G$ on $\mathbf{H}^{3}$ can be regarded as simplicial. Hence for any finite index subgroup $N$ of $G, \operatorname{Dim}\left(H_{*}\left(\mathbf{H}^{3} / G ; \mathbf{R}\right)\right) \leq \operatorname{Dim}\left(H_{*}\left(\mathbf{H}^{3} / N ; \mathbf{R}\right)\right)$ by Theorem 2. Since $\mathbf{H}^{3} / G$ is homeomorphic to $S^{3}-\{k$ points $\}$ (see the proof of Theorem $1), k-1 \leq \operatorname{Dim}\left(H_{2}\left(\mathbf{H}^{3} / N ; \mathbf{R}\right)\right)$ and $\mathbf{H}^{3} / N$ has at least $k$ cusps.

REMARK 3. The proof of Theorem 1 can be used to obtain some information about the rank of normal t.f.i. subgroups of some polyhedral groups. For example, the tetrahedral groups $T_{1}, T_{2}, T_{3}, T_{4}, T_{5}, T_{7}, T_{8}, T_{9}, T^{1}, T^{5}, T^{10}$ and $T^{21}$ do not contain a normal t.f.i. subgroup of rank 2 (see $\$ 3$ for the notation). And the Picard group can only contain normal t.f.i. subgroups of rank at least 3 .

In $\S 2$, we prove Theorems 1 and 2, and in $\S 3$, we verify Remark 3.

I would like to thank A. Brunner, T. Fournelle, N. Wielenberg and the referee of the original manuscript for many useful suggestions and D. Solitar for inviting me to York University where the proof of Theorem 1 was found. 
2.

2.1. Theorem 1 follows immediately from the next two lemmas which will be proven later.

LEMMA 1. Let $N$ be a normal t.f.i. subgroup of the polyhedral group $G$ associated to a polyhedron $P$. If the rank of $N$ is $n(>0)$, then there exists a nontrivial homomorphism $\Gamma$ from $G$ to $\mathrm{GL}(n, \mathbf{Z})$ such that the image of $\Gamma$ is finite and the rank of $H_{1}\left(\mathbf{H}^{3} / K ; \mathbf{Z}\right)$ is $n$, where $K$ is the kernel of $\Gamma$.

Lemma 2. Let $P$ be a polyhedron with one or no ideal vertex. If $P$ is not exceptional and $K$ is an index 2 subgroup of the polyhedral group $G$ associated to $P$, then $H_{1}\left(\mathbf{H}^{3} / K ; \mathbf{Z}\right)=0$. (The proof of the lemma shows that $\mathbf{H}^{3} / K$ is homeomorphic to a 3-sphere with finitely many points removed but we do not need this.)

We now turn to the proof of Theorem 1. Suppose that the polyhedral group $G$ is associated to polyhedron $P$. If $P$ has more than one ideal vertex, then the theorem holds trivially by Remark 2 .

We assume that $P$ has one or no ideal vertex. If $G$ contains a normal t.f.i. subgroup of rank 1 , then by Lemma 1 there exists a nontrivial homomorphism $\Gamma$ : $G \rightarrow \mathrm{GL}(1, \mathbf{Z})$ such that $H_{1}\left(\mathbf{H}^{3} / K ; \mathbf{Z}\right)$ has rank 1 , where $K$ is the kernel of $\Gamma . K$ is an index 2 subgroup of $G$ since $G L(1, Z) \cong Z_{2} . P$ is exceptional because if not, $H_{1}\left(\mathbf{H}^{3} / K ; \mathbf{Z}\right)=0$ by Lemma 2 . This completes the proof of the theorem.

Proof of Lemma 1. Suppose that $P$ has $k$ ideal vertices. Let $x$ be a point in the interior of $P$. We regard $P$ as the cone of ( $\partial P \cup$ (ideal vertices $\})$ with respect to $x$ using the geodesics starting at $x$ and ending at a point in $\partial P$ or at an ideal vertex. Suppose that $F_{i}, i=1,2, \ldots, m$, are the faces of $P$ and $R_{i}$ is the reflection of $\mathbf{H}^{3}$ in $F_{i}$. Let $x * F_{i}(\subset P)$ be the cone of $\left(F_{i} \cup\right.$ ideal vertices in $\left.\left.F_{i}\right\}\right)$ with respect to $x$. Define $Q=P \cup_{1 \leq i \leq m} R_{i}\left(x * F_{i}\right)$. All the faces of $Q$ are triangular and an edge of $P$ is an edge of $Q$ but $Q$ has some new edges. It is easy to see that $Q$ is a fundamental domain of $G$ and $Q$ has $k$ ideal vertices (see [5] for details).

It is convenient (especially for the proof of Lemma 2) to identify $Q$ as the following space. Let $\mathbf{R}_{\infty}^{3}$ be the Euclidean 3-space $\mathbf{R}^{3}$ compactified by adding a point at infinity so that $\mathbf{R}_{\infty}^{3}$ is homeomorphic to the 3 -sphere. We identify $P$ with the complement of the unit open ball $\stackrel{\circ}{B}^{3}$ centered at the origin in $\mathbf{R}_{\infty}^{3}$ with $k$ points removed from the boundary $\left(S^{2}=\partial B^{3}\right)$ corresponding to the ideal vertices of $P$.

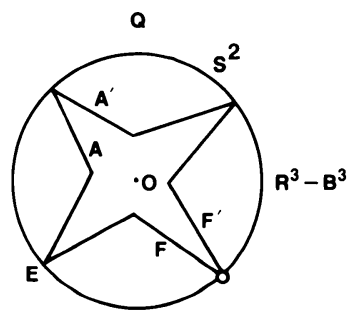

FIGURE 1 
The edges of $P$ give naturally a partition of $S^{2}$. We regard $B^{3}$ as the cone of $S^{2}$ with respect to $O$, the origin of $\mathbf{R}^{3}$. For each edge $E$ of $P$, cut $\mathbf{R}_{\infty}^{3}$ open along $O * E$ and remove the $k$ points from $S^{2}$ corresponding to the ideal vertices. The space we have constructed is clearly homeomorphic to $Q$. A two-dimensional picture of $Q$ is given in Figure 1.

$G$ induces a pairwise identification of the faces of $Q$ and $\mathbf{H}^{3} / G$, the orbit space of $G$, is homeomorphic to $Q /$ (the face identification). Recall that an element $g$ of $G$ identifies faces of $Q$ if $g(Q) \cap Q$ is a union of faces of $Q$. Let $F$ be a face of $Q$. Then there exists a unique edge $E$ of $P$ (an edge of $Q$ which is also an edge of $P$ will be called an old edge) such that $F$ is one of the two copies of $O * E$ along which $\mathbf{R}_{\infty}^{3}$ is cut open. Denote the other copy by $F^{\prime}$. There exists an element (a rotation along $E$ ) of $G$ which identifies $F$ with $F^{\prime}$. Therefore, the identification of faces of $Q$ induced by $G$ is exactly the reverse of the cutting we used to get $Q$ from $\mathbf{R}_{\infty}^{3}-\{k$ points . This shows that $\mathbf{H}^{3} / G$ is homeomorphic to $S^{3}-\{k$ points $\}$, in particular $H_{1}\left(\mathbf{H}^{3} / G ; \mathbf{Z}\right)=0$. We will omit the coefficients of a homology if they are $\mathbf{Z}$.

Let $H=G / N$ and $X=\mathbf{H}^{3} / N$. The action of $G$ on $\mathbf{H}^{3}$ induces a discontinuous action of $H$ on $X$ such that the following diagram commutes [3].

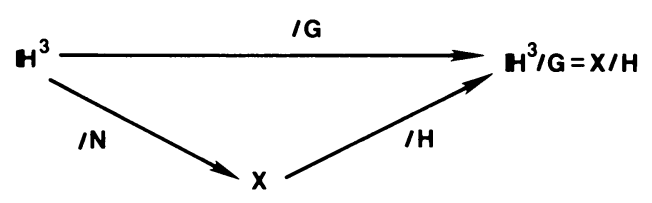

Since $N \cong \pi_{1}(X), H_{1}(X) /$ Tor $\cong n \mathbf{Z}$ (the direct sum of $n$ copies of $\mathbf{Z}$ ), where $H_{1}(X) /$ Tor is $H_{1}(X)$ modulo the torsion subgroup.

We now state a theorem which is crucial for this paper. A proof of the theorem can be found in $\S 3$ of [3].

THEOREM A. If a finite group $H$ acts on a space $X$ (simplicial complex) simplicially, then $H_{*}(X ; \Lambda)^{H}$ is isomorphic to $H_{*}(X / H ; \Lambda)$, where $\Lambda$ is a field of characteristic 0 and $H_{*}(X ; \Lambda)^{H}$ is the subgroup of fixed points of the induced action of $H$ on the homology. The homology groups in the theorem may be replaced with the homology groups over $\mathbf{Z}$ modulo the torsion subgroups.

We return to the proof of Lemma 1 . Since we may regard that $H$ acts on $X$ simplicially and $H$ is finite, Theorem A implies that

$$
\left(H_{1}(X) / \text { Tor }\right)^{H} \cong H_{1}(X / H) / \text { Tor }=H_{1}\left(\mathbf{H}^{3} / G\right)=0 \text {. }
$$

Let $\psi: H \rightarrow \mathrm{GL}(n, \mathbf{Z}) \cong \operatorname{Aut}\left(H_{1}(X) /\right.$ Tor $)$ be the homomorphism induced by the action of $H$ on $H_{1}(X) /$ Tor. The image of $\psi$ is a nontrivial finite subgroup of $\operatorname{GL}(n, \mathbf{Z})$. Define $\Gamma$ as the quotient map followed by $\psi, \Gamma: G \rightarrow H \rightarrow \operatorname{GL}(n, \mathbf{Z})$. Let $L$ and $K$ be the kernel of $\psi$ and $\Gamma$, respectively. $H / L \cong G / K$ and we have the following commutative diagram. 


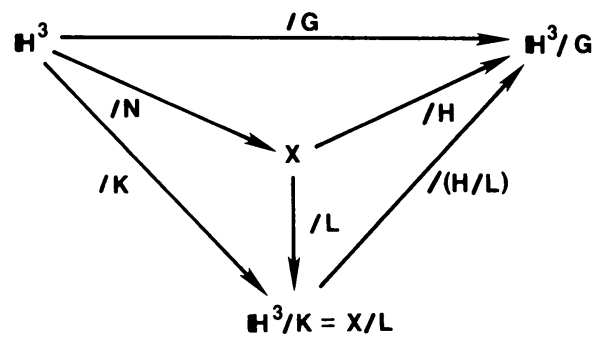

By Theorem A,

$$
\left(H_{1}(X) / \text { Tor }\right)^{L} \cong H_{1}\left(\mathbf{H}^{3} / K\right) / \text { Tor }
$$

and by construction, $\left(H_{1}(X) / \text { Tor }\right)^{L} \cong n \mathbf{Z}$. Therefore, $H_{1}\left(\mathbf{H}^{3} / K\right) /$ Tor $\cong n \mathbf{Z}$.

Proof OF LemMa 2. Let $\{1, b\}$ be a system of right coset representatives of $K$ in $G$ such that $Q$ is disjoint from $b(Q)$, where $Q$ is defined in the proof of Lemma 1 . Let $R=b(Q)$ and $Y=Q \cup R . Y$ is a fundamental domain (nonconnected ) of $K$ and $K$ induces a pairwise face identification of $Y$. Let $I$ be the subset of $K$ of elements identifying faces of $Y$. Then $Y / I \cong \mathbf{H}^{3} / K$. Let $I_{1}$ and $I_{3}$ be the subsets of $I$ of elements identifying the faces of $Q$ and $R$, respectively, among themselves, and let $I_{2}$ be the subset of elements identifying faces of $Q$ with faces of $R$. Notice that $I_{2}$ is not empty.

Suppose that $i \in I_{1}$ and $i$ identifies face $A$ of $Q$ with face $B$. There exists $g \in G$ such that $g$ identifies $A$ with $A^{\prime}$, where $A^{\prime}$ is the unique face of $Q$ sharing an old edge with $A$. The elements $i$ and $g$ of $G$ identify $A$ with $B$ and $A^{\prime}$, respectively. This implies that $B=A^{\prime}$ and $i=g$. Therefore, elements of $I_{1}$ glue back some of the faces of $Q$ the way they were before the cutting. $Q / I_{1}$ is homeomorphic to a closed 3-ball with $k$ points removed, where $k(=0$ or 1$)$ is the number of ideal vertices of $P$. A removed point is either in the interior or on the boundary of the 3-ball. We also point out that the face structure of $Q$ induces a face structure (or rather a partition) on $Q / I_{1}$.

Suppose that $i \in I_{1}$ and $i$ identifies $A$ with $A^{\prime}$ by a rotation along the common old edge $E$. Then $b i b^{-1}$ identifies $b(A)$ with $b\left(A^{\prime}\right)$ by a rotation along $b(E)$. Since $K$ is normal (recall that $K$ is of index 2 in $G$ ) $b i b^{-1} \in K$ and thus $b i b^{-1} \in I_{3}$. Therefore, $b$ induces a homeomorphism from $Q / I_{1}$ to $R / I_{3}$ respecting the face structure.

We now need to investigate the face structure of $Q / I_{1}$. Let $v$ be an old vertex of $Q$ (that is, $v$ is a vertex of $P$ ). Notice that a face of $Q$ contains exactly two old vertices and a third vertex coming from the cone point. There are eight faces of $Q$ sharing $v$ if $v$ is an ideal vertex of type $(2,2,2,2)$ and there are six faces otherwise. Suppose that $v$ is an ideal vertex of type $(2,2,2,2)$ and $r_{1}, r_{2}, r_{3}$ and $r_{4}$ are the standard generators of the stabilizer of $v$ read in a proper order. Then $r_{1} r_{2} r_{3} r_{4}=1$ is a defining relation of $G$. $K$ contains at least one of the four generators of the stabilizer since $P$ is not exceptional but then $K$ contains at least two of them because of the above relation. Therefore, if $v$ is still a vertex of $Q / I_{1}$, then there are two or four faces sharing $v$ in $Q / I_{1}$. If $v$ is not an ideal vertex of type $(2,2,2,2)$ and $v$ is a vertex 

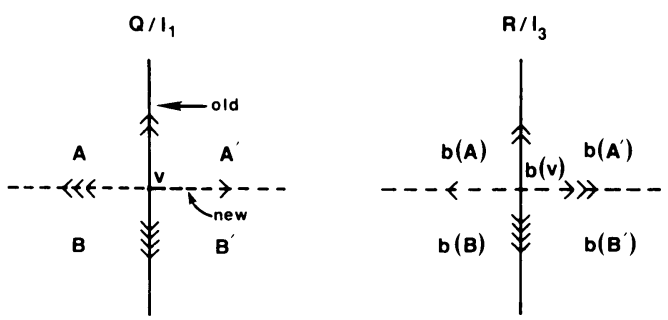

Figure 2

of $Q / I_{1}$, then we can conclude the same regarding the number of faces sharing $v$ in $Q / I_{1}$ using the defining relation $r_{1} r_{2} r_{3}=1$, where $r_{1}, r_{2}, r_{3}$ are generators of the stabilizer of $v$.

We now compute $H_{1}\left(\mathbf{H}^{3} / K\right)$ by the Mayer-Vietoris sequence. Denote $Q^{\prime}=$ $\left(Q / I_{1} \cup \partial R / I_{3}\right) / I_{2}, \quad R^{\prime}=\left(R / I_{3} \cup \partial Q / I_{1}\right) / I_{2}$ and $S=\left(\partial Q / I_{1} \cup \partial R / I_{3}\right) / I_{2}$. Then $\mathbf{H}^{3} / K \cong Y / I=Q^{\prime} \cup R^{\prime}$, where the union is done along $S=Q^{\prime} \cap R^{\prime}$. Since $S$ is connected, $H_{1}\left(\mathbf{H}^{3} / K ; \mathbf{Z}\right)=0$ if $H_{1}\left(Q^{\prime}\right)=H_{1}\left(R^{\prime}\right)=0$. We verify the last identities by showing that $Q^{\prime}$ and $R^{\prime}$ are homeomorphic to $Q / I_{1}$ and $R / I_{3}$, respectively.

We first observe that $Q^{\prime}$ is $Q / I_{1}$ with some of the edges of $Q / I_{1}$ identified among themselves. This follows from the fact that $I_{2}$ identifies faces of $Q / I_{1}$ with those of $R / I_{3}$ in a one-to-one fashion but $I_{2}$ may identify two distinct edges, say $E_{1}$ and $E_{2}$, of $Q / I_{1}$ to the same edge of $R / I_{3}$. If this happens, we must identify $E_{1}$ with $E_{2}$ to get $Q^{\prime}$ from $Q / I_{1}$. We claim that $I_{2}$ does not induce any identification of edges of $Q / I_{1}$ and the same for $R / I_{3}$.

To see this, let $A$ be a face of $Q / I_{1}$ and $A^{\prime}$ the copy of $A$ obtained from the cutting. Suppose that $g \in I_{2}$ identifies $A$ with a face of $R / I_{3}$. Then $b^{-1} g$ identifies $A$ with a face of $Q / I_{1}$. Obviously, $b^{-1} g(A)=A^{\prime}$ and $b^{-1} g$ is the rotation $r$ along the common edge of $A$ with $A^{\prime}$. Therefore, $g$ identifies $A$ with $b\left(A^{\prime}\right)$ and $g=b r$. Suppose that $E$ is an edge of $Q / I_{1}$. E contains at least one old vertex, say $v$, of $Q / I_{1}$. Now one needs to check several cases to see that $E$ is not identified with another edge in $Q / I_{1}$ through $I_{2}$. Figure 2 shows one of the cases when $v$ is contained in four faces in $Q / I_{1}$. The verification of the other cases is left to the reader. (It may be interesting to see that our claim is false if there are six or eight faces sharing $v$.)

Finally, the argument given above shows that $I_{2}$ defines a homeomorphism between $\partial\left(Q / I_{1}\right)$ and $\partial\left(R / I_{3}\right)$. Therefore, $\mathbf{H}^{3} / K \cong Q / I_{1} \cup R / I_{3}$ is homeomorphic to $S^{3}$ with $k$ points removed, where $k=0,1$ or 2 .

2.2. We prove Theorem 2. Let $M$ be a normal subgroup of $N_{1}$ contained in $N_{2}$ as a finite index subgroup. The existence of $M$ is easy to see. The following diagram commutes.

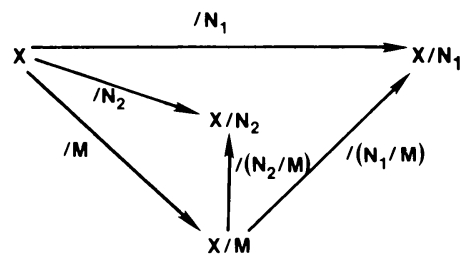


By Theorem $\mathrm{A}, H_{*}(X / M ; \mathbf{R})^{N_{1} / M} \cong H_{*}\left(X / N_{1} ; \mathbf{R}\right)$, where $\mathbf{R}$ is the set of real numbers. On the other hand,

and

$$
H_{*}(X / M ; \mathbf{R})^{N_{2} / M} \cong H_{*}\left(X / N_{2} ; \mathbf{R}\right)
$$

$$
H_{*}(X / M ; \mathbf{R})^{N_{1} / M} \subset H_{*}(X / M ; \mathbf{R})^{N_{2} / M}
$$

since the action of $N_{1} / M$ on $X / M$ induces the action of $N_{2} / M$. This completes the proof of the theorem.

3.

3.1. For positive integers $\lambda_{1}, \lambda_{2}, \lambda_{3}, \mu_{1}, \mu_{2}, \mu_{3}$, the tetrahedral group $T\left(\lambda_{1}, \lambda_{2}, \lambda_{3} ; \mu_{1}, \mu_{2}, \mu_{3}\right)$ is the group associated to the tetrahedron in Figure 3.

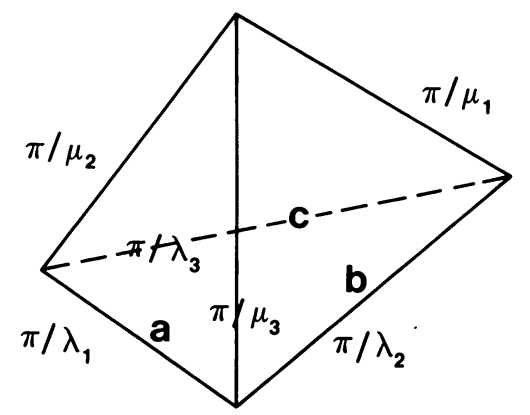

Figure 3

Using the cone construction in 2.1 and Poincaré's theorem [7], we can find a presentation of the group:

$$
\left\langle a, b, c ; a^{\lambda_{1}}=b^{\lambda_{2}}=c^{\lambda_{3}}=(a b)^{\mu_{3}}=(a c)^{\mu_{2}}=\left(b^{-1} c\right)^{\mu_{1}}=1\right\rangle .
$$

Here $a, b$ and $c$ are the rotations along the edges with dihedral angles $2 \pi / \lambda_{1}, 2 \pi / \lambda_{2}$ and $2 \pi / \lambda_{3}$, respectively.

We list all 32 tetrahedral groups [11]. Groups with superscripts have ideal vertices and groups with subscripts do not.

$$
\begin{array}{lll}
T_{1}(2,2,3 ; 3,5,2), & T_{2}(2,2,3 ; 2,5,3), & T_{3}(2,2,4 ; 2,3,5), \\
T_{4}(2,2,5 ; 2,3,5), & T_{5}(2,3,3 ; 2,3,4), & T_{6}(2,3,4 ; 2,3,4), \\
T_{7}(2,3,3 ; 2,3,5), & T_{8}(2,3,4 ; 2,3,5), & T_{9}(2,3,5 ; 2,3,5)
\end{array}
$$

and

$$
\begin{array}{lll}
T^{1}(3,2,2 ; 6,2,3), & T^{2}(2,2,3 ; 2,6,3), & T^{3}(3,2,2 ; 4,2,4), \\
T^{4}(4,2,2 ; 6,2,3), & T^{5}(5,2,2 ; 6,2,3), & T^{6}(6,2,2 ; 6,2,3), \\
T^{7}(4,2,2 ; 4,2,4), & T^{8}(2,3,3 ; 2,6,3), & T^{9}(3,2,4 ; 3,2,6), \\
T^{10}(6,2,3 ; 5,2,3), & T^{11}(3,2,6 ; 3,2,6), & T^{12}(2,3,3 ; 2,4,4), \\
T^{13}(4,2,3 ; 4,2,4), & T^{14}(4,2,4 ; 4,2,4), & T^{15}(3,2,2 ; 2,3,6), \\
T^{16}(3,2,2 ; 2,4,4), & T^{17}(4,2,2 ; 2,4,4), & T^{18}(3,2,3 ; 3,3,3), \\
T^{19}(3,2,2 ; 3,3,3), & T^{20}(4,2,2 ; 3,3,3), & T^{21}(5,2,2 ; 3,3,3), \\
T^{22}(6,2,2 ; 3,3,3), & T^{23}(3,3,3 ; 3,3,3) . &
\end{array}
$$




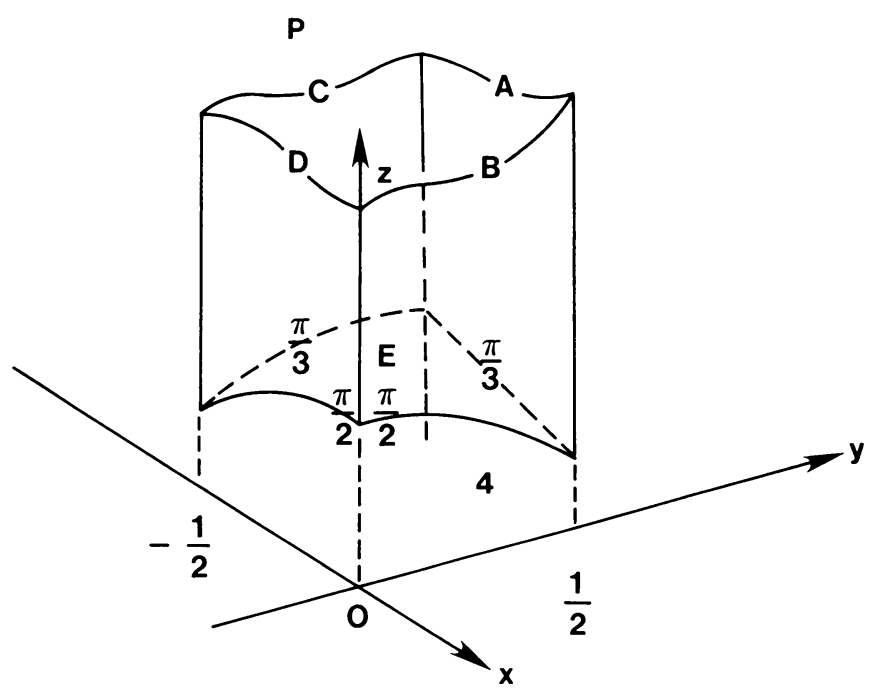

Figure 4

The only homomorphism from one of $T_{1}, T_{2}, T_{4}, T_{5}, T_{7}, T_{8}, T_{9}, T^{10}$ and $T^{21}$ into $\mathrm{GL}(2, \mathbf{Z})$ is trivial. This can be seen easily from the presentation of these groups and the fact that elements of finite order in $\operatorname{GL}(2, \mathbf{Z})$ have order $2,3,4$, or 6. Furthermore, there are only three elements of order 2 and one element of order 3, 4, or 6 up to conjugation [8]. By Lemma 1, these groups cannot contain a normal subgroup of rank 2. For $T_{3}, T^{1}$, or $T^{5}$ it can be shown that the image of a homomorphism from one of these groups into $\operatorname{GL}(2, \mathbf{Z})$ is either trivial or isomorphic to $\mathbf{Z}_{2}$. By Lemma 2, these groups do not contain a normal t.f.i. subgroup of rank 2.

3.2. We now pick the polyhedral group $G$ associated to the pentahedron $P$ in Figure 4. $P=\left\{(x+y i+z j):-\frac{1}{2} \leq x \leq 0,0 \leq y \leq \frac{1}{2}, z \geq \sqrt{1-x^{2}-y^{2}}\right\} . P$ is not exceptional because each index 2 group of $G$ contains one of the standard generators of the stabilizer of the ideal vertex.

The group is known as $\operatorname{PSL}(2, \mathbf{Z}(i))$ or the Picard group [10] and its torsion-free subgroups of index 12 and 24 are classified in [4]. We show that the Picard group can only contain a normal t.f.i. subgroup of rank at least 3 . This is the greatest lower bound since the Borromean link group (rank 3) occurs as a normal t.f.i. subgroup of index 24 [4].

Let $e$ be the reflection of $\mathbf{H}^{3}$ in the face $E$ of $P$, where $E$ is given as the bottom face of $P$ in Figure 4. Let $Q=P \cup e(P) . Q$ is a fundamental domain of $G$. The faces of $Q$ are drawn spread out on the complex plane in Figure 5. $(x+y i ; d)$ denotes the hemisphere in $\mathbf{H}^{3}$ centered at $x+y i$ with radius $d$. The face identification induced by $G$ is also given in Figure 5. A face denoted by a letter is identified to the face denoted by the same letter with a prime such that the edges represented by the arrows in the interior of faces are matched respecting the direction. 

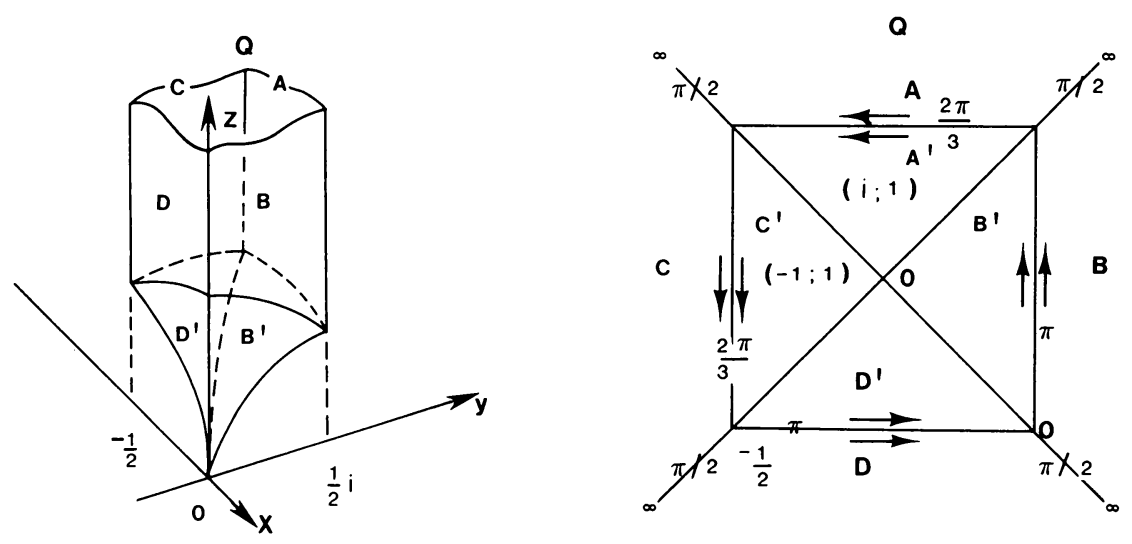

\section{FIGURE 5}

If we denote $a$ for the element of $G$ identifying $A$ to $A^{\prime}$ and similarly for $b, c$ and $d$, then by [7], $G$ has a presentation,

$$
\begin{gathered}
\left\langle a, b, c, d: a^{3}=c^{3}=b^{2}=d^{2}=(a b)^{2}=\left(a^{-1} c\right)^{2}=(c d)^{2}=(b d)^{2}=1\right\rangle, \\
a=\left(\begin{array}{cc}
0 & i \\
i & 1
\end{array}\right), \quad b=\left(\begin{array}{cc}
0 & 1 \\
-1 & 0
\end{array}\right), \quad c=\left(\begin{array}{cc}
0 & 1 \\
-1 & -1
\end{array}\right) \text { and } d=\left(\begin{array}{ll}
0 & i \\
i & 0
\end{array}\right) .
\end{gathered}
$$

Using the information about $\operatorname{GL}(2, \mathbf{Z})$ described in 3.1, one can show that if $\Gamma$ : $G \rightarrow \mathrm{GL}(2, \mathbf{Z})$ is a nontrivial homomorphism and $I$ is its image, then there are five possibilities as listed below up to the isomorphisms of $G$ and conjugations of $\mathrm{GL}(2, \mathbf{Z})$.

(1) $I \cong \mathbf{Z}_{2} ; \Gamma(a)=\Gamma(c)=\Gamma(d)=0$ and $\Gamma(b)=1$, where $\mathbf{Z}_{2}=\{1,0\}$.

(2) $I \cong \mathbf{Z}_{2} ; \Gamma(a)=\Gamma(c)=0$ and $\Gamma(b)=\Gamma(d)=1$.

(3) $I \cong \mathbf{Z}_{2} \oplus \mathbf{Z}_{2} ; \Gamma(a)=\Gamma(c)=(0,0), \Gamma(b)=(1,0)$ and $\Gamma(d)=(0,1)$.

(4) $I \cong S_{3}=\left\langle\alpha, \beta: \alpha^{3}=\beta^{2}=(\alpha \beta)^{2}=1\right\rangle ; \Gamma(a)=\Gamma(c)=\alpha$ and $\Gamma(b)=\Gamma(d)$ $=\beta$.

(5) $I \cong\left\langle\alpha, \beta, \delta: \alpha^{3}=\beta^{2}=\delta^{2}=(\alpha \beta)^{2}=(\beta \delta)^{2}=1\right\rangle ; \Gamma(a)=\Gamma(c)=\alpha, \Gamma(b)=$ $\beta$ and $\Gamma(d)=\delta$.

If $G$ has a normal t.f.i. subgroup of rank 2, then $H_{1}\left(\mathbf{H}^{3} / K\right) /$ Tor $\cong 2 \mathrm{Z}$ in one of the above five cases by Lemma 1 , where $K$ is the kernel of $\Gamma$. Lemma 2 rules out cases (1) and (2). To compute $H_{1}\left(\mathrm{H}^{3} / K\right)$ for the remaining cases, one may use the Reidemeister-Schreier subgroup theorem to get a presentation of $K$ and then use [2]. But the geometric method [6] is used here which gives us $\mathbf{H}^{3} / K$ directly from a right coset representation of $K$ in $G$. Fundamental domains $Q_{3}, Q_{4}$ and $Q_{5}$ of $K$ for the cases (3), (4) and (5) are given in Figures 6, 7, and 8, respectively. $Q_{3}=Q \cup b(Q) \cup$ $d(Q) \cup b d(Q), Q_{4}=\cup_{0 \leq i \leq 2,0 \leq j \leq 1} b^{j} a^{i}(Q)$ and $Q_{5}=Q_{4} \cup d\left(Q_{4}\right)$. The ideal vertices are 0 and $\infty$ for $Q_{3}, 0, i$ and $\infty$ for $Q_{4}$, and $0, i,-i$ and $\infty$ for $Q_{5}$. The figures also show the face identification of the fundamental domain induced by $K$ in each case. $\mathbf{H}^{3} / K$ is homeomorphic to an $S^{3}$ with one point removed in case (3) and three points removed in cases (4) and (5). Therefore, $H_{1}\left(\mathbf{H}^{3} / K\right)=0$.

This completes the proof that the Picard group can only contain normal t.f.i. subgroup of rank at least 3 . 


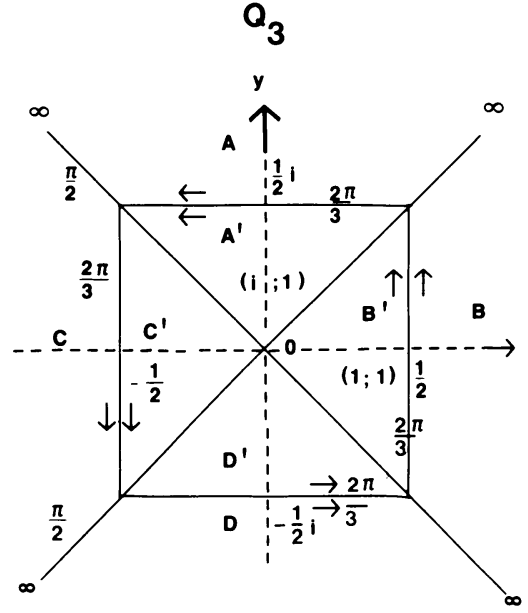

FIGURE 6

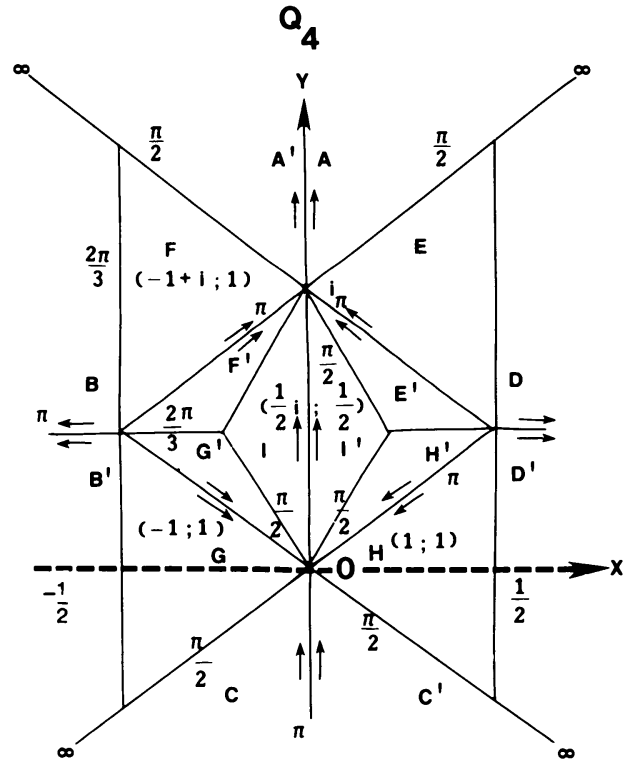

FIGURE 7 


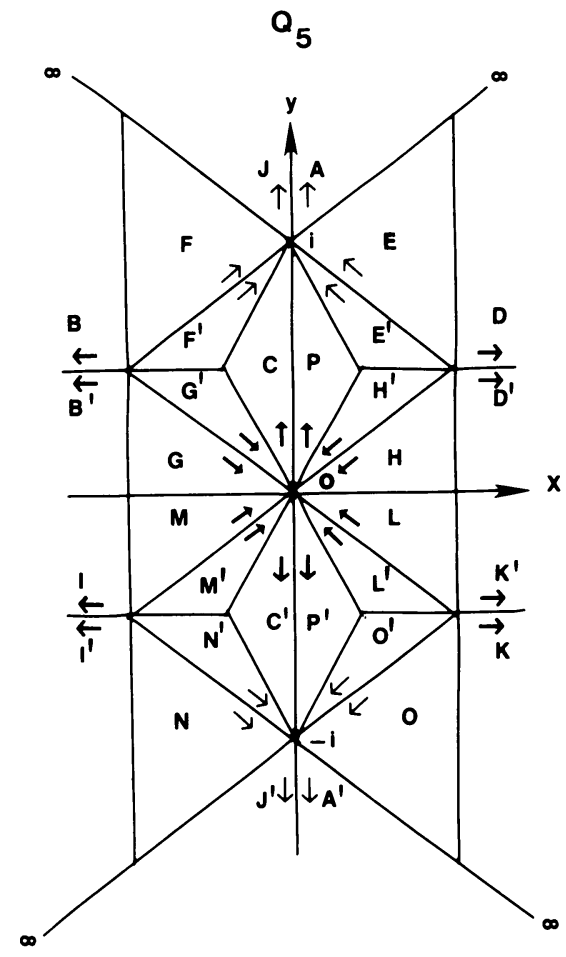

Figure 8

\section{REFERENCES}

1. E. Andreev, On convex polyhedra of finite volume in Lobacevskii spaces, Mat. Sb. 83(125) (1970); English transl., Math. USSR Sb. 12(1970), 225-259.

2. M. Armstrong, The fundamental group of the orbit space of a discontinuous group, Proc. Cambridge Philos. Soc. 64 (1968), 299-301.

3. G. Bredon, Introduction to compact transformation groups, Academic Press, New York and London, 1972.

4. A. Brunner, M. Frame, Y. Lee and N. Wielenberg, Classifying torsion-free subgroups of Picard group, Trans. Amer. Math. Soc. 282 (1984), 205-235.

5. A. Brunner, Y. Lee and N. Wielenbreg, Polyhedral groups and graph amalgamation products, Topology Appl. (to appear).

6. Y. Lee, A geometric method for presenting subgroups of discrete groups, Topology Appl. 18 (1984), 179-195.

7. B. Maskit, On Poincaré's theorem for fundamental polygons, Adv. in Math. 7 (1971), 219-230.

8. S. Meskin, Periodic automorphisms of the two-generator free group, Lecture Notes in Math., vol. 372 , Springer-Verlag, Berlin and New York, 1974, pp. 494-498.

9. R. Riley, A quadratic parabolic group, Math. Proc. Cambridge Philos. Soc. 77 (1975), 281-288.

10. R. Swan, Generators and relations for certain special linear groups, Adv. in Math. 6 (1971), 1-71.

11. W. Thurston, The geometry and topology of 3-manifolds, Princeton Univ. Press, Princeton, N. J., 1982.

12. F. Waldhausen, On irreducible 3-manifolds which are sufficiently large, Ann. of Math. (2) 87 (1968), $56-88$.

13. N. Wielenberg, The structure of certain subgroups of the Picard group, Math. Proc. Cambridge Philos. Soc. 84 (1978), 427-436.

Department of Mathematics, University of Wisconsin - Parkside, Kenosha, Wisconsin 53141 